\title{
"NOS GUSTARÍA QUE NOS ACOMPAÑASEN EN NUESTRAS DECISIONES”. ALGUNAS CUESTIONES QUE PREOCUPAN A PERSONAS ADULTAS CON DISCAPACIDAD INTELECTUAL
}

\section{"We would like them to accompany us in our decisions". Some issues that concern adults with intellectual disability}

\author{
Susana Rojas PERNIA \\ Universidad de Cantabria. Departamento de Educación. Avda. los Castros, s/n. 39005 Santander \\ (Cantabria) \\ rojass@unican.es \\ Ignacio Haya SALmón \\ Universidad de Cantabria. Departamento de Educación. Avda. los Castros, s/n. 39005 Santander \\ (Cantabria)
}

Recepción: 8 de marzo de 2017

Aceptación definitiva: 1 de septiembre de 2017

RESUMEN: El artículo presenta los principales resultados de una investigación cualitativa que tiene por objeto indagar en la experiencia subjetiva de 16 personas con discapacidad intelectual en torno a esferas vitales como la formación, el trabajo, la vivienda, las relaciones de pareja o la familia. La recogida de datos se lleva a cabo mediante entrevistas semiestructuradas y en profundidad. El proceso de investigación presta especial atención a los aspectos éticos que aquí son explicitados como elementos vertebradores en la toma de decisiones metodológicas. Con todo ello, accedemos a los relatos personales de los participantes para su posterior análisis. Los resultados se organizan en torno a cuatro ideas que visibilizan las circunstancias que rodean en la actualidad a algunas personas adultas con discapacidad intelectual, recurrentes en los relatos de los participantes. Así, 
“NOS GUSTARÍA QUE NOS ACOMPAÑASEN EN NUESTRAS DECISIONES”. ALGUNAS CUESTIONES QUE PREOCUPAN A PERSONAS ADULTAS CON DISCAPACIDAD INTELECTUAL SUSANA ROJAS PERNIA E IGNACIO HAYA SALMÓN

se abordan asuntos como: la presión de la crisis económica, la distancia entre los deseos y los proyectos personales, el "siempre en preparación" y la condición de "ciudadano amputado". La discusión de resultados refleja los avances y cambios sociales y políticos de estos últimos años. Aun con todo, como los participantes señalan, hay obstáculos que hacen que decisiones como independizarse en pareja o formar una familia sean ilusiones todavía difíciles de materializar.

Palabras Clave: discapacidad intelectual; ciudadanía; control social; investigación inclusiva; apoyos personales y sociales.

AвSTRACT: This article presents the main results of a qualitative research that aims to explore the subjective experience of 16 people with intellectual disability around different vital spheres such as training, work, housing, relationships, or family. Data collection is carried out through semi-structured and in-depth interviews. The research process pays special attention to the ethical aspects that are explicitly shown as key elements in the decision-making process. Thus, we have access to the personal statements of the participants for further analysis. The results are organized around four topics that make visible the circumstances that currently surround some adults with intellectual disabilities due to this statements are recurrent in their stories. Issues such as the pressure of the economic crisis, the distance between desires and plans, always in preparation and the status of "citizen amputee" are addressed. The discussion of results reflects the social and political changes of recent years. However, all the participants identify some obstacles that make independent living or starting a family are still difficult illusions to materialize.

KEYWORDS: people with intellectual disability; citizenship; social control; inclusive research; personal and social supports.

\section{Introducción}

$\mathrm{E}$

N LOS ÚlTimOS AÑos se han producido cambios importantes en las respuestas a las personas con discapacidad desde el ámbito social, político, educativo o investigador. Sin duda, la aprobación de la Convención Internacional de los Derechos de las personas con discapacidad (ONU, 2006) es expresión de todo ese conjunto de cambios. En este sentido, algunas de las decisiones adoptadas concretamente en el contexto español se reflejan en experiencias e investigaciones que dan cuenta de situaciones en las que personas con discapacidad intelectual viven en entornos comunitarios con el apoyo de otras personas (Fernández Cordero, 2013; Trias, 2015; Puyalto, 2016), representan intereses colectivos a través de grupos de autodefensa (Pérez Gil, 2007; Tamarit y Espejo, 2013; Claro y Pecino, 2015) o participan activamente en trabajos de investigación (Haya, Rojas y Lázaro-Visa, 2014; Pallisera y Puyalto, 2014; Fullana, Pallisera, Vilà y Puyalto, 2016). La transformación en la manera de abordar las políticas educativas y sociales y la propia investigación ligada a las personas con discapacidad producida desde finales de los ochenta han venido acompañadas de un mayor reconocimiento social. 
“NOS GUSTARÍA QUE NOS ACOMPAÑASEN EN NUESTRAS DECISIONES”. ALGUNAS CUESTIONES QUE PREOCUPAN A PERSONAS ADULTAS CON DISCAPACIDAD INTELECTUAL SUSANA ROJAS PERNIA E IGNACIO HAYA SALMÓN

No obstante, y a pesar de los logros alcanzados, es posible señalar que estos son parciales. Tal y como analizan algunos informes (Colectivo IOÉ, 2012; Rodríguez, 2012), es posible afirmar que el nivel de estudios de las personas con discapacidad ha mejorado en los últimos años o que la mayoría de los niños con discapacidad entre 6 y 15 años en España están escolarizados en centros ordinarios. Sin embargo, solo dos tercios del alumnado con discapacidad alcanza a los 16 años el nivel de estudios de primaria (Rodríguez, 2012) y en el curso 2015/16 la cifra de alumnado en régimen de educación especial aumentó respecto al año anterior $(1,8 \%)$ (MEC, 2015). En lo que se refiere al empleo, esta es la actividad principal para el $28 \%$ de las personas con discapacidad, pero más de dos terceras partes en edad laboral no trabajan. La presencia fuera de los Centros Especiales de Empleo es, como señala Toboso-Martín (2013), esporádica y en trabajos de baja cualificación. De igual modo, aunque en los últimos años ha crecido la demanda del "direct payment" por parte de las personas con discapacidad, no se ha producido una mayor inversión en la asistencia personal, especialmente en algunas comunidades autónomas, lo que no facilita la desinstitucionalización y la promoción de la vida en la comunidad (Romañach, 2011).

Por tanto, la educación, el empleo o el acceso a una vivienda propia siguen siendo un asunto pendiente para muchas personas con discapacidad, como lo es el hecho de poder decidir en torno a las cuestiones que les importan y afectan sin ser sustituidas por familiares o profesionales (Tamarit y Espejo, 2013; Etxeberría, 2016). Los trabajos o investigaciones que documentan la promoción de la autogestión o la autodefensa en personas con discapacidad intelectual ejemplifican la orientación adoptada desde las entidades particulares y su contribución en el reconocimiento de ciudadanos de pleno derecho, pero al mismo tiempo expresan las limitaciones sociales para reconocer, respetar y promover el derecho a la autogestión. Tal y como analiza y recoge el informe Independent but not alone (Inclusion International, 2014), las personas con discapacidad intelectual ven limitadas las oportunidades para tomar decisiones formales o jurídicas, pero también para decidir sobre asuntos de su vida personal o comunitaria, sobre cuestiones económicas y de salud.

El trabajo de investigación que presentamos explora las cuestiones anteriormente planteadas desde la experiencia subjetiva de los participantes, como ya hicieran trabajos previos (Booth y Booth, 1994; Shakespeare, Gillespie-Sells y Dominic, 1996; Goodley, 2000). Concretamente, y con el propósito general de conocer y visibilizar los asuntos que son importantes en la vida de un grupo de personas con discapacidad intelectual, se abordan tópicos como la formación, el trabajo, el ocio o las relaciones personales.

El artículo que a continuación se desarrolla expone, en primer lugar, algunas cuestiones metodológicas nucleares relacionadas con las consideraciones éticas en el proceso de investigación; en segundo lugar, analiza cuatro ideas que emergen de los relatos de los participantes y que están condicionando las decisiones que algunas personas con discapacidad intelectual pueden o no tomar en sus vidas. Esas cuatro ideas son: la presión de la crisis económica, la distancia entre los deseos y proyectos personales, el “siempre en preparación” y la condición de “ciudadanía amputada”. 
“NOS GUSTARÍA QUE NOS ACOMPAÑASEN EN NUESTRAS DECISIONES”. ALGUNAS CUESTIONES QUE PREOCUPAN A PERSONAS ADULTAS CON DISCAPACIDAD INTELECTUAL SUSANA ROJAS PERNIA E IGNACIO HAYA SALMÓN

\section{Método}

\subsection{Diseño de la investigación}

Desde el punto de vista metodológico, el estudio que presentamos se reconoce en el paradigma cualitativo de investigación (Denzin y Lincoln, 2012), con una clara inspiración en los planteamientos que sostiene la investigación inclusiva (Williams, 2000; Gilbert, 2004; Nind, 2014; Walmsley, 2004a, 2004b). Esta apuesta emerge de la necesidad de alcanzar una mayor comprensión de las experiencias subjetivas en las áreas vitales ya mencionadas de las personas que participaron en el estudio y de la exigencia de garantizar las condiciones que hiciesen posible un mayor control por parte de los participantes en decisiones nucleares del estudio. Con todo ello, se inició el estudio que nos ocupa, proponiendo a las personas con discapacidad intelectual un tiempo para dialogar sobre su experiencia vital y sus proyectos de futuro. Los relatos permiten una aproximación a la complejidad y sutileza de su experiencia y facilitan una aproximación al contexto social y a la cultura en la que esta se desarrolla (Webster y Mertova, 2007).

\subsection{Participantes}

En la investigación participan 16 adultos (6 mujeres y 10 hombres) entre 18 y 39 años que viven en dos comunidades autónomas españolas (Tabla 1). En el momento en el que las entrevistas fueron realizadas, la mayoría estaba en algún proceso de formación para el empleo $(n=12)$ y cuatro de ellas tenían empleo en empresas ordinarias. De estas últimas, una vivía de manera independiente y las otras tres en el hogar familiar.

Todas las personas utilizaban el lenguaje verbal como principal recurso comunicativo.

\begin{tabular}{|l|c|c|c|c|c|c|}
\hline \multicolumn{7}{|c|}{ Tabla 1. Información sobre los participantes en la investigación } \\
\hline $\begin{array}{l}\text { PARTICIPANTE } \\
\text { (NOMBRE FICTICIO) }\end{array}$ & EDAD & SEXO & ACTIVIDAD PRINCIPAL & $\begin{array}{c}\text { NIVEL EDUCATIVO } \\
\text { FINALIZADO }\end{array}$ & VIVIENDA & $\begin{array}{c}\text { RELACIÓN } \\
\text { PAREJA }\end{array}$ \\
\hline Ismael & 21 & $\mathrm{H}$ & Trabajo EO $1 / 2$ jornada & Primaria & Hogar parental & NO \\
\hline Gabriel & 30 & $\mathrm{H}$ & Trabajo EO & ESO & Hogar parental & NO \\
\hline Joan & 29 & $\mathrm{H}$ & Formación & Primaria & Hogar parental & NO \\
\hline Zelatan & 18 & $\mathrm{H}$ & Formación & Primaria & Hogar parental & NO \\
\hline Mónica & 22 & $\mathrm{M}$ & Trabajo EO $1 / 2$ jornada & Primaria & & \\
\hline Maria & 23 & $\mathrm{M}$ & Formación & Primaria & Hogar parental & NO \\
\hline Tesa & 30 & $\mathrm{M}$ & Formación & ESO & Hogar parental & NO \\
\hline Eric & 30 & $\mathrm{H}$ & Empleo protegido & Primaria & Hogar parental & NO \\
\hline Rosa & 32 & $\mathrm{M}$ & Empleo protegido & Primaria & Independiente & SÍ \\
\hline Oriol & 39 & $\mathrm{H}$ & Empleo protegido & Primaria & Independiente & SÍ \\
\hline Diego & 33 & $\mathrm{H}$ & Empleo protegido & Primaria & Hogar parental & SÍ \\
\hline
\end{tabular}


“NOS GUSTARÍA QUE NOS ACOMPAÑASEN EN NUESTRAS DECISIONES”. ALGUNAS CUESTIONES QUE PREOCUPAN A PERSONAS ADULTAS CON DISCAPACIDAD INTELECTUAL SUSANA ROJAS PERNIA E IGNACIO HAYA SALMÓN

Tabla 1. Información sobre los participantes en la investigación

\begin{tabular}{|c|c|c|c|c|c|c|}
\hline $\begin{array}{l}\text { PARTICIPANTE } \\
\text { (NOMBRE FICTICIO) }\end{array}$ & EDAD & SEXO & ACTIVIDAD PRINCIPAL & $\begin{array}{l}\text { Nivel EDUCATIVO } \\
\text { FINALIZADO }\end{array}$ & VIVIENDA & $\begin{array}{c}\text { RELACIÓN } \\
\text { PAREJA }\end{array}$ \\
\hline Mia & 37 & M & Empleo protegido & Primaria & Serv. residencial & $\mathrm{NO}$ \\
\hline Luna & 37 & $\mathrm{M}$ & Empleo protegido & Primaria & Serv. residencial & SÍ \\
\hline Gustavo & & $\mathrm{H}$ & Empleo protegido & Primaria & Hogar parental & $\mathrm{NO}$ \\
\hline Javier & 30 & $\mathrm{H}$ & Trabajo EO & ESO & Hogar parental & $\mathrm{NO}$ \\
\hline Asier & 18 & $\mathrm{H}$ & Formación & Primaria & Serv. residencial & $\mathrm{NO}$ \\
\hline
\end{tabular}

Empresa Ordinaria (EO).

\subsection{Instrumentos}

La recogida de información se lleva a cabo mediante entrevistas semiestructuradas en profundidad (Kvale, 2011). Las preguntas fueron agrupadas en torno a seis grandes bloques temáticos: 1) Autopresentación, en la que el participante facilitaba información sobre sí mismo al investigador. 2) Vivienda, englobaba una serie de cuestiones sobre el entorno vital o hábitat del entrevistado, desde el punto de vista físico, personal y convivencial. 3) Formación y empleo, agrupaba interrogantes acerca de la trayectoria formativa y la situación laboral. 4) Ocio, incluía cuestiones relacionadas con las iniciativas de entretenimiento y el modo en que el entrevistado gestiona su tiempo libre. 5) Relaciones sociales, recogía diferentes preguntas acerca del entramado social y las relaciones de amistad que el entrevistado disfruta y, por último, 6) Relaciones afectivas/pareja, con la que pretendíamos aproximarnos a la esfera de relaciones más íntimas y personales de los participantes.

En la propia entrevista se incorporó otra estrategia en la que el lenguaje visual cobraba más importancia. Específicamente, fueron utilizadas un conjunto de imágenes con las que profundizar (Booth y Booth, 1994, 2003; Hammersley y Atkinson, 2009) sobre uno de los tópicos, el de las relaciones afectivas y sexuales. Estas imágenes, ilustraciones o fotografías, iban de lo concreto - una pareja con clara actitud afectiva u otra con un bebé en brazos, por ejemplo- a lo abstracto -laberinto u objetos de la vida cotidiana-. Se solicitó a los participantes que escogiesen una imagen que mejor representase su idea de pareja para describir qué inquietudes y necesidades compartía en torno a este asunto: qué busca en una pareja, qué importancia tiene en su vida o si le gustaría o no vivir una relación amorosa.

\subsection{Procedimiento}

El acceso a los participantes se realizó a través de profesionales que desde el ámbito asociativo apoyan el desarrollo de programas que promueven la autodeterminación y la autodefensa de personas con discapacidad intelectual. De este modo, se satisfacen los dos criterios nucleares de selección de participantes en la investigación: personas 
“NOS GUSTARÍA QUE NOS ACOMPAÑASEN EN NUESTRAS DECISIONES”. ALGUNAS CUESTIONES QUE PREOCUPAN A PERSONAS ADULTAS CON DISCAPACIDAD INTELECTUAL SUSANA ROJAS PERNIA E IGNACIO HAYA SALMÓN

con discapacidad intelectual que tienen o han tenido contacto con grupos de trabajo en el ámbito de la autodeterminación y la autodefensa. Tras ese primer contacto, se informó individualmente de los propósitos de la investigación y se solicitó autorización a las personas con discapacidad intelectual y sus representantes legales.

Las dieciséis entrevistas fueron grabadas en audio, previa autorización expresa de los participantes, y posteriormente trascritas. La información fue completada con otros datos que el entrevistador recogió durante las sesiones (p. e. los silencios, los momentos de incomodidad o las emociones contenidas).

El análisis de los datos se realizó a partir de un proceso de codificación temática (Gibbs, 2012). Para ello, se identifican fragmentos de los textos con unidad temática (unidades de significado) a los que asigna un código. El catálogo de categorías y códigos se elaboró en base a procesos de análisis deductivo-inductivo, es decir, procesos de ida y vuelta del marco teórico de la investigación a los relatos construidos por los participantes. Así emerge un catálogo provisional de códigos que sería empleado para codificar cuatro entrevistas - dos cada uno de los investigadores por separado- para su posterior discusión y adecuación hasta obtener el catálogo definitivo. Apoyados en el programa informático MAXQDA 11 (Kuckartz, 2007) se codificaron las 16 entrevistas. Finalizado el proceso de codificación temática, un tercer investigador actuó como "juez externo", en calidad de experto cuya trayectoria de investigación se caracteriza por una larga experiencia en estudios sobre discapacidad desde el paradigma cualitativo. Se comprobó que las unidades de significado seleccionadas se adecuaban a los códigos descritos en el catálogo. Así, de los veinticinco fragmentos facilitados al juez externo, veintitrés resultaron coincidentes en los códigos asignados por los investigadores ( $92 \%$ de coincidencia). Con todo ello, se establece un total de siete categorías -seis de ellas coincidentes con los ejes temáticos señalados y en torno a los que se organiza la entrevista, y otra para organizar los datos personales y sociodemográficos- que a su vez contienen una serie de códigos. Como se muestra en el ejemplo (Tabla 2) los códigos adscritos a cada categoría organizan las declaraciones de los informantes en torno a los proyectos, deseos y las barreras y apoyos que se identifican en los relatos de los participantes.

Tabla 2. Ejemplo de Categorías y códigos empleados en el análisis de los datos

\begin{tabular}{cl}
\hline & \multicolumn{1}{c}{ CATEgORÍA: Formación y EMPLEO } \\
\hline Nombre código & \multicolumn{1}{c}{ CONTENido } \\
\hline Descripción Form.-Emp. & $\begin{array}{l}\text { Descripción del tipo de empleo/formación que desarrolla el infor- } \\
\text { mante (lugar de trabajo/estudio, itinerario formativo, retribucio- } \\
\text { nes, etc.). }\end{array}$ \\
\hline Barreras Form.-Emp. & $\begin{array}{l}\text { Declaraciones sobre limitaciones o dificultades percibidas por el } \\
\text { informante (personales o sociales-contexto) para desarrollar una } \\
\text { actividad laboral/formativa. }\end{array}$ \\
\hline Apoyos Form.-Emp. & $\begin{array}{l}\text { Declaraciones sobre ayudas percibidas por el informante (perso- } \\
\text { nales o sociales-contexto) para desarrollar una actividad laboral/ } \\
\text { formativa. }\end{array}$ \\
\hline
\end{tabular}

(C) Ediciones Universidad de Salamanca / CC BY-NC-ND

Siglo Cero, vol. 48 (3), n. ${ }^{\circ}$ 263, 2017, julio-septiembre, pp. 55-72

$$
-60-
$$


“NOS GUSTARÍA QUE NOS ACOMPAÑASEN EN NUESTRAS DECISIONES”. ALGUNAS CUESTIONES QUE PREOCUPAN A PERSONAS ADULTAS CON DISCAPACIDAD INTELECTUAL SUSANA ROJAS PERNIA E IGNACIO HAYA SALMÓN

Tabla 2. Ejemplo de Categorías y códigos empleados en el análisis de los datos

\begin{tabular}{cl}
\hline Nombre CÓDIgo & \multicolumn{1}{c}{ CATEgoría: Formación y EMPLeO } \\
\hline Proyecto Form.-Emp. & $\begin{array}{l}\text { Declaraciones en las que el informante comparte cómo planifica y } \\
\text { se produce la toma de decisiones relacionada con un itinerario de } \\
\text { formación y empleo que el participante considera valioso para sí } \\
\text { mismo. Hace referencia a lo real, acciones en desarrollo. }\end{array}$ \\
\hline $\begin{array}{l}\text { Declaraciones en las que se manifiesta algo que no se tiene y que } \\
\text { se ansía tener, que no se ha logrado aún o que no se ha concreta- } \\
\text { do en un proyecto formativo o de empleo por el momento. Hace } \\
\text { referencia a los anhelos del participante, sin que necesariamente se } \\
\text { haya trazado un plan de actuación para satisfacerlos. }\end{array}$ \\
\hline
\end{tabular}

El análisis comparativo de todos los textos agrupados bajo el mismo código facilita la organización de los principales resultados del trabajo de investigación que son presentados más adelante. Asimismo, dicho análisis sirvió para organizar la información en la devolución a los participantes. En dos sesiones de trabajo con estos, a las que asistieron también los profesionales que facilitaron el acceso a las personas entrevistadas, se discutió sobre los datos obtenidos en las entrevistas. Para la presentación de resultados en las reuniones se recurrió también al sistema de "lectura fácil" (García Muñoz, 2011).

\section{Aspectos éticos de la investigación}

La investigación tomó como referente el Código de Buenas Prácticas del CSIC (2011). Desde el inicio del trabajo de investigación se planteó la importancia que tiene que las personas conocieran la naturaleza del estudio, su finalidad y los tópicos que definían la entrevista. Antes de obtener el consentimiento informado, se hizo explícito el compromiso de los investigadores de mantener el anonimato de los participantes y suprimir la información que, una vez finalizada la entrevista o tras su revisión, desearan retirar. Del mismo modo, se informó a los participantes que se emplearían nombres ficticios en la difusión y comunicación de los resultados de la investigación. La decisión final de participar estuvo supeditada en algún caso a la autorización expresa de un tutor legal.

Para asegurar la comprensión de todas estas cuestiones, se elaboró un documento de apoyo en el que se recogían las mismas de acuerdo con el sistema de "lectura fácil". Este documento se empleó únicamente con las personas que manifestaron su interés por utilizarlo.

La entrevista fue concebida como un recurso flexible de manera que se pudiera, en función de las circunstancias, introducir cambios ad hoc. Los tópicos centrales de la entrevista fueron planteados por los investigadores, pero la profundidad con la que fue abordada cada una de las cuestiones varió en función de la persona participante. Se insistió individualmente en el derecho a escoger las preguntas a las que no deseara 
“NOS GUSTARÍA QUE NOS ACOMPAÑASEN EN NUESTRAS DECISIONES”. ALGUNAS CUESTIONES QUE PREOCUPAN A PERSONAS ADULTAS CON DISCAPACIDAD INTELECTUAL

contestar y nuestro compromiso de no perseverar. El siguiente fragmento ejemplifica esto último:

Entrevistador: Me estabas diciendo lo que era importante en una relación de pareja, ¿tú crees que las relaciones sexuales son muy importantes en una relación de pareja?

Oriol: No te puedo contestar a eso.

Entrevistador: ¿No quieres o...?

Oriol: No quiero contestarte a eso.

Entrevistador: No quieres contestar, vale (Oriol, 39 años).

Evidentemente, asumir el control sobre el contenido de las entrevistas trasciende al mero hecho de responder o no a las preguntas formuladas por los entrevistadores. La elección de un formato de entrevista semiestructurada permite que el guion de la conversación entre entrevistador/entrevistado se construya, en parte, a medida que esta se desarrolla y que aspectos valorados como importantes por los participantes sean incorporados al trabajo de investigación.

Durante el desarrollo de la entrevista y la técnica de la foto se integraron las decisiones de los participantes y se aseguraron las adaptaciones que permitiesen, como hicieran otros trabajos anteriores, que ninguno de los jóvenes fuese excluido de la investigación (Aldridge, 2007; Rojas, Susinos y Calvo, 2013). El siguiente cuadro recoge las decisiones metodológicas que fueron adoptadas:

Tabla 3. Decisiones vinculadas con el carácter inclusivo de la investigación

EN LA PRESENTACIÓN DEL ESTUDIO

- Explicitar el sentido y finalidad del trabajo.

- Anticipar tópicos presentes en la entrevista, mostrar algunos ejemplos de las preguntas.

- Clarificar los compromisos (confidencialidad y anonimato) adquiridos por los investigadores.

- Solicitar autorización para grabar en audio las entrevistas.

- Facilitar apoyo visual de la información. Elaboración de un guion de presentación en "lectura fácil".

- Preguntar sobre las condiciones de la entrevista para garantizar que la persona las conocía y compartía.

EN EL DESARROLLO DE LAS ENTREVISTAS

- Facilitar la elección al participante del lugar y momento en el que se desarrolla la recogida de información.

- Garantizar que el entrevistado no tiene que abordar tópicos sobre los que no desea hablar.

- Flexibilizar en el diseño y formulación de las preguntas de la entrevista.

- Valorar la importancia que tiene el lenguaje no verbal.

- Utilizar diversidad de canales y soportes comunicativos.

- Incluir los contenidos sugeridos y valorados por los participantes durante el desarrollo de la investigación.

- Incluir preguntas que inviten a la reflexión sobre la relevancia del estudio para las personas con discapacidad y la comunidad en su conjunto.

- Incorporar preguntas que evaluasen las técnicas utilizadas en la recogida de datos.

EN EL TRABAJO CON LOS DATOS

- Ofrecer la oportunidad a los participantes de retirar cualquier información que deseen.

- Facilitar el contenido de la entrevista al participante que lo solicite. 
“NOS GUSTARÍA QUE NOS ACOMPAÑASEN EN NUESTRAS DECISIONES”. ALGUNAS CUESTIONES QUE PREOCUPAN A PERSONAS ADULTAS CON DISCAPACIDAD INTELECTUAL

Tabla 3. Decisiones vinculadas con el carácter inclusivo de la investigación

EN LA DEVOLUCIÓN DE RESULTADOS

- Facilitar los resultados a todos los participantes en un formato accesible.

- Recoger las dudas, preocupaciones y demandas que las personas plantean.

La investigación, concebida desde el primer momento como un espacio desde el que apoyar y empoderar a los participantes (Walmsley, 2004b), se tradujo en un reconocimiento de la persona que dialoga, tanto de su subjetividad como del valor de sus aportaciones. El proceso seguido permitió, tal y como muestra el siguiente ejemplo, reflexionar sobre el valor personal y de transformación social que podía tener el estudio del que formaban parte:

Entrevistador: ¿Qué te ha parecido la entrevista?

Asier: Pues me ha parecido muy bien, ¡ha estado bien!

Entrevistador: ¿Por qué?

Asier: Porque está bien contárselo a alguien, porque sé que voy a estar seguro de la persona que viene a hacerte una entrevista y sé que te va a ayudar bastante.

Entrevistador: ¿A qué crees que me puede ayudar a mí esta entrevista, o a otras personas?

Asier: Pues a la experiencia que yo te he contado, y también para ver si se puede... pues las cosas que sean malas pues hacerlas, intentar que sean mejores y las que son mejores pues intentar que sean mejores todavía.

Entrevistador: Pues eso es lo que intentaremos, intentar compartir con otra gente...

Asier: Sí, las experiencias.

Entrevistador: ... lo que funciona y lo que no, para que cada uno pueda llevar la vida que quiere (Asier, 18 años).

Para los investigadores, la valoración final de la entrevista que realizaron los participantes permitió introducir mejoras respecto al guion inicial, así como adquirir un conocimiento más situado de las herramientas diseñadas.

\section{Resultados}

Del análisis de los datos emergen diversidad de declaraciones sobre las condiciones que rodean a las personas con discapacidad intelectual que participaron en la investigación, así como sobre los deseos y proyectos que se sitúan en el centro de sus preocupaciones. Estas declaraciones sirven como punto de partida para introducir la presentación de los resultados, organizada en torno a cuatro grandes epígrafes que nos permiten ofrecer una panorámica sobre dichas condiciones, en términos de barreras y ayudas, que convergen en las distintas dimensiones o esferas vitales exploradas.

En primer lugar, bajo el epígrafe denominado "La presión de la crisis económica" se ilustra en qué medida las condiciones económicas, personales y sociales interfieren 
“NOS GUSTARÍA QUE NOS ACOMPAÑASEN EN NUESTRAS DECISIONES”. ALGUNAS CUESTIONES QUE PREOCUPAN A PERSONAS ADULTAS CON DISCAPACIDAD INTELECTUAL SUSANA ROJAS PERNIA E IGNACIO HAYA SALMÓN

en la vida de los participantes. De hecho, y de manera recurrente, once de las dieciséis personas identifican o aluden a determinadas cuestiones económicas como principales obstáculos para desarrollarse satisfactoriamente en cada una de las esferas vitales exploradas. En segundo lugar, bajo el epígrafe "La distancia entre los deseos y los proyectos" son puestas en diálogo las barreras y apoyos identificados con los proyectos y deseos vitales de los participantes. Así, este epígrafe permite ilustrar cuáles son los proyectos y deseos de los participantes relacionados con las distintas esferas vitales exploradas y qué barreras reconocen ellos mismos como las más significativas. El tercer epígrafe se centra en una situación compartida por todos los participantes sin excepción: el control que perciben sobre sus vidas en las distintas esferas vitales está fuertemente condicionado por la visión de quienes les rodean como sujetos que han de estar “siempre en preparación”. Finalmente, el cuarto y último epígrafe, la condición de "ciudadanía amputada”, pretende desvelar las principales dificultades identificadas en los relatos de los participantes que, presentes en las distintas esferas vitales distintas, interfieren en la vivencia de una ciudadanía plena. En cada uno de los epígrafes se introducen, a modo de ejemplo, algunos fragmentos representativos de los resultados que serán discutidos posteriormente.

\subsection{La presión de la crisis económica}

La grave situación económica que afecta a España es una cuestión nuclear para la mayoría de los entrevistados. En sus discursos, las restricciones económicas les obligan a pensar de qué manera organizar mejor los escasos recursos económicos que tienen y continuar posponiendo algunos de sus deseos: ["Yo comparo, si cuesta esto, si esto cuesta digamos 10 euros, no lo compro. O si cuesta por ejemplo, si cuesta 60 tampoco, tampoco me lo puedo permitir, porque tengo que llegar... ahora me tengo que organizar" (Oriol, 39 años)] .

Los jóvenes se refieren a dificultades económicas que se tornan obstáculos para 1) hacer frente a necesidades básicas [“[...] mis padres me tienen que ayudar con los gastos [...] mi sueldo es para los gastos de la hipoteca, para todo el suma y sigue. Si no es por mis padres que me dan algo de dinero... (estaría ahogada)” (Rita, 32 años)]; 2) lograr una mayor autonomía personal [“¡Sí!, yo siempre he querido (vivir) sola, pero si no, con amigas (y no lo he intentado porque no puedo económicamente), igual que sacarme el carnet de conducir, como no hay dinero...” (Tesa, 30 años)]; y 3) contribuir al bienestar familiar [“(el trabajo me hacía sentir) bien porque ayudaba a mi familia, a mis padres, a mi hermana. Bueno, como traía dinero a casa, no cobraba mucho pero bueno... algo. Íbamos mejor porque es que ahora sin trabajo y como están las cosas...” (Joan, 29 años)].

En consecuencia, para algunas personas es inevitable definir como inviables o arriesgadas decisiones tales como independizarse del hogar familiar o irse a vivir en pareja, como señalaba uno de los jóvenes cuando se le pregunta si cree que para algunas personas es más difícil independizarse del núcleo familiar que para otras: 
“NOS GUSTARÍA QUE NOS ACOMPAÑASEN EN NUESTRAS DECISIONES”. ALGUNAS CUESTIONES QUE PREOCUPAN A PERSONAS ADULTAS CON DISCAPACIDAD INTELECTUAL SUSANA ROJAS PERNIA E IGNACIO HAYA SALMÓN

[...] ahora mismo, en la actualidad, creo que tanto para las personas con discapacidad como sin discapacidad, irse de casa, con la crisis económica que hay actualmente, es muy difícil. Yo creo que sí, porque sale mucho en TV que ahora, gente con 28 y 29 años está viviendo con sus padres, porque no pueden, aunque no sea pagando una hipoteca o, aunque sea alquilar un piso, depende del sueldo que ganes, ¡no puedes! No puedes ni pagar un alquiler y encima, si tienes trabajo, ganas poco y si no tienes trabajo, todavía menos, no puedes permitirte ni pagar un alquiler. ¡Yo creo que es imposible! (Gabriel, 30 años).

Los entrevistados coincidieron en afirmar que para las personas con discapacidad los obstáculos para independizarse son mayores que para el resto y esta dificultad es aún mayor para algunas personas con discapacidad intelectual. Concretamente, entre los obstáculos los participantes se refieren a limitaciones personales (falta de preparación o de capacidades) ["Yo soy una persona discapacitada mentalmente y bueno, por mí, yo puedo vivir (de manera independiente)... pero claro, personas de discapacidad más fuerte, que tienen más, no sé” (Zelatan, 18 años) y otros a cuestiones relacionadas con la accesibilidad ["Yo creo que no, que les costará más a las personas con discapacidad, supongo [...] Por las adaptaciones que puedan necesitar y todo, más que nada” (María, 23 años)]. Con todo, es la dependencia económica el principal obstáculo con el que se encuentran y que interfiere en el desarrollo de sus proyectos vitales. Además, la crisis económica fue precisamente la que dejó sin trabajo a alguno de los participantes ["Por temas económicos no había... por la crisis, todo eso que no, no tenían dinero y por algún lado tenían que recortar. Me tocó a mí” (Joan, 29 años)].

Los discursos de los jóvenes denuncian que sus escasos medios económicos restringen las posibilidades de control personal sobre sus vidas y sobre lo que les gustaría hacer en un futuro ["Como te he dicho antes, hasta que no tenga nada él (su pareja), yo no me voy a arriesgar, no. Y me encantaría ser madre, pero no sé si voy a poder porque como tengo ovarios poliquísticos [...] pero hasta que no vea algo fijo, yo no me voy a arriesgar (Rita, 32 años)].

Sin embargo, para otras personas ese malestar no parece estar presente. Para cinco de los jóvenes, el tema económico no asoma en ningún momento en sus narraciones. Se trata de personas que viven en el hogar familiar o en una alternativa residencial, que explicitan la posibilidad de vivir algún día en pareja o de formar una familia, pero que no se han planteado nunca dar ese paso o si lo han hecho, como reconoce una de las entrevistadas, es improbable ["Él y yo lo estamos deseando pero ya no se puede, no sé, lo veo un poco difícil. Porque claro, él sípodría, él me ha dicho a mi que sí, pero está mi tutor, mi tutor que me ha dicho que no" (Luna, 37 años)].

\subsection{La distancia entre los deseos y los proyectos vitales}

Los participantes expresan diversidad de anhelos en distintas esferas vitales. Esos deseos se relacionan para algunos con el acceso a un trabajo ["Lo que más me gustaría es trabajar, como todo el mundo" (Zelatan, 18 años)], con la idea de encontrar una 
“NOS GUSTARÍA QUE NOS ACOMPAÑASEN EN NUESTRAS DECISIONES”. ALGUNAS CUESTIONES QUE PREOCUPAN A PERSONAS ADULTAS CON DISCAPACIDAD INTELECTUAL SUSANA ROJAS PERNIA E IGNACIO HAYA SALMÓN

pareja ["Me gustaría encontrar una pareja, que no tengo" (Gabriel, 33 años)] o irse a vivir con ella [“(Lo que más me gustaría) la pareja, vivir con mi pareja” (Luna, 37 años)], con la oportunidad de ampliar el grupo de relaciones ["Por eso te digo que es lo que me falta, hacer más vida social” (Gabriel, 33 años), con la posibilidad de formar una familia ["Pues sería que yo tuviera un niño" (Ismael, 21 años) o de independizarse del hogar parental ["Sí, me gustaría independizarme” (Joan, 29 años). Algunas de esas afirmaciones aparecen desde el primer momento, cuando les preguntamos por algo que no tienen y desearían tener y son una constante a lo largo de la conversación. Así pues, los deseos como expresiones regidas socialmente, son tantos y tan amplios como participantes.

Sin embargo, la posibilidad de que estos deseos se conviertan en proyectos vitales, en un plan más o menos trazado de ideas y acciones para hacer realidad sus deseos, es en muchas ocasiones difuso, como relata uno de los participantes:

Alberto: Algún día si pudiese me gustaría tener con mi pareja un poco de familia.

Entrevistador: Te gustaría formar una familia.

Alberto: Sí.

Entrevistador: ¿Y ella?

Alberto: Pues no lo sé, igual sí, igual no, no sé, no hemos hablado de esto, de formar una familia, de momento no hemos hablado (33 años).

En otras ocasiones, la distancia entre los deseos y proyectos vitales resulta insalvable debido al control que terceras personas ejercen sobre las vidas de las personas con discapacidad intelectual:

Mia: No puedo vivir sola. Sé cocinar, sé planchar, sé hacer de todo... se puede decir que el día que yo me case, al marido no le falta...

Entrevistador: Pero, tú me dices "no puedo vivir sola” ... pero ahora me estás diciendo que "el día que me case voy a tener al marido bien cuidado".

Mia: Si me caso.

Entrevistador: Bueno, si te casas... entonces...

Mia: ¡Y si puedo! ¡Y si puedo! ¡Si me dejan!

Entrevistador: ¿Si puedes?, ¿de qué depende que puedas?

Mia: La decisión no la puedo tomar yo (37 años).

A las barreras sociales cabe añadir las que se vinculan con aspectos socioeconómicos y que constituyen, como se señaló, un obstáculo relevante para la realización de sus deseos vitales.

A pesar de lo anterior, algunas personas sienten que tienen el control sobre lo que irá ocurriendo. Afirman que los proyectos irán tomando forma con el tiempo, en función de lo que vaya aconteciendo y de futuras experiencias. En palabras de uno de los participantes, "Mi madre siempre me dice, ¡tú vas a conseguir lo que tú quieras! [...]. Tú no esperes nunca” (Zelatan, 18 años). 
“NOS GUSTARÍA QUE NOS ACOMPAÑASEN EN NUESTRAS DECISIONES”. ALGUNAS CUESTIONES QUE PREOCUPAN A PERSONAS ADULTAS CON DISCAPACIDAD INTELECTUAL SUSANA ROJAS PERNIA E IGNACIO HAYA SALMÓN

\subsection{El "siempre en preparación"}

La afirmación anterior - “tú no esperes nunca”- tropieza con lo que parece ser una constante en la vida de todos los participantes. En líneas generales es posible referenciar un proceso de entrenamiento que se extiende a todos los ámbitos en la vida de las personas con discapacidad intelectual y que las obliga a prepararse para obtener un puesto de trabajo, para independizarse familiarmente, para disfrutar de un ocio normalizado, para tener una pareja o parar formar una familia.

El acceso a los programas preparatorios presupone que las personas con discapacidad intelectual reúnen las capacidades y habilidades necesarias para lograr los objetivos que se persiguen desde los programas en los que participan, pero necesitan desarrollarlas. Como describe María, después de dos años en formación laboral, en la actualidad sigue aprendiendo nuevas habilidades que entiende que son importantes:

[...] ahora estoy haciendo solo competencias laborales, autoconocimiento y habilidades sociales. Y un viernes al mes vengo a la sesión de todos juntos. Bien, está muy bien todo, la verdad. Sí, sí, yo creo que sí (que son cosas que necesito) (23 años).

Se define como un tiempo de preparación necesario para acceder más tarde en condiciones óptimas, en esta ocasión, a un puesto de trabajo.

Sería posible opinar que este proceso es similar al que sigue la mayoría de la población de forma habitual. No obstante, es importante señalar que la preparación no se circunscribe al contexto formativo y laboral y tampoco asegura a la persona el acceso a aquello por lo que se inscribió en el programa una vez este finaliza, aun cuando demuestre disponer de las habilidades requeridas.

En este contexto, es inevitable plantear que las “oportunidades” son para algunas personas con discapacidad intelectual una hoja de doble filo. Si no se logra lo previsto, la responsabilidad será suya ya que no era realmente capaz. No se cuestiona la organización de los servicios (ocio, formación para el empleo o preparación para la vida independiente) o las oportunidades reales que las personas tienen para lograr algunos de sus deseos. Asimismo, porque todo lo anterior refuerza la idea de déficit que constriñe a muchas personas con discapacidad y justifica el control que otras personas ejercen sobre ellas en las distintas áreas de la vida: "No, ni soy de bares ni, hombre, de discoteca tampoco soy, pero no, no me dejan tampoco, (mis padres) no me dejan" (Joan, 29 años). Como este y el siguiente relato muestran, ello conduce a una situación de indefensión que las personas interiorizan, aceptando lo que ha sido y será su relación con otras personas:

Entrevistador: María, ¿tú has pensado alguna vez en...jno digo para hacerlo ya pero, ¿has pensado alguna vez "me tendré que ir de casa, me iré de casa"?

María: No, en principio no, porque como no veo mucho y todo... pues en principio no.

Entrevistador: ¿Y qué te gustaría, irte sola, irte con amigos, irte con una pareja?

María: No lo sé todavía.

Entrevistador: ¿Tú qué crees que pensarían tus padres si les dijeras, “papás me quiero independizar”? 


\section{“NOS GUSTARÍA QUE NOS ACOMPAÑASEN EN NUESTRAS DECISIONES”. ALGUNAS CUESTIONES QUE PREOCUPAN A PERSONAS ADULTAS CON DISCAPACIDAD INTELECTUAL SUSANA ROJAS PERNIA E IGNACIO HAYA SALMÓN}

María: No lo sé, yo creo que se lo tomarían bien. Yo creo que sí, que se lo tomarían bien, los primeros días supongo que querrían venir a controlar un poco y todo.

Entrevistador: ¿Qué crees que querrían controlar?

María: Un poco todo en general, supongo, ya como costumbre más que nada (23 años).

\subsection{La condición de "ciudadanía amputada"}

Todos los participantes reflexionaron en torno a las posibilidades y limitaciones de su condición de ciudadanos. En algunas declaraciones se emplea el término "derechos" para denunciar la condición de "ciudadanos amputados", en tanto que son privados de opciones fundamentales, y reivindicar el reconocimiento a ejercer el control de su propia vida, principalmente, en esferas tan privadas e íntimas, como la vida familiar y la vida en pareja, "[...] somos gente que también tenemos derecho a tener una familia, derecho a tener pareja, derecho a vivir con la pareja” (Alberto, 33 años).

Las afirmaciones, aunque expresadas de formas distintas, se sitúan en el nivel de denuncia que muestra el siguiente ejemplo:

Javier: Algo que no tengo y que me gustaría... pues hombre, algo que no tengo y me gustaría, pues yo diría, tener una vida normal. Bueno vida normal, me refiero, yo portarme... a ver, cómo lo explico, para mí es complicado explicar eso. [...] Saberme comportar en, digamos, saber tener el día de mañana una vida normal, como todo hijo de vecino, todo en la calle, pues normal, pues una persona con una casa, un trabajo, su vida de ocio, su coche, su lo que sea, pues tener que sí, tener tu discapacidad, pero fuera de lo demás pues tener tus cosas (30 años).

Algunos de los jóvenes matizan que sus limitaciones personales suponen un obstáculo en algunas situaciones, pero son las barreras sociales (actitud de rechazo, la indiferencia ante determinadas políticas y prácticas), explícitas u ocultas, las que generan mayor marginación. Como plantea una de las entrevistadas, la pérdida de control sobre derechos básicos abarca cuestiones que llegan hasta la posibilidad de decidir libremente sobre su participación en la investigación:

Mia: $R$. vive sola, jvive sola! $R$. se lo puede permitir, no tiene una, no nos permiten, no está tutelada, que es a eso a lo que voy. [...] Como abora, yo be venido aquí a contarte todo, yo tengo que firmar papeles que tú... y solo ha sido una entrevista, imira tú!” (37 años).

Finalmente, para algunos de los participantes la condición de ciudadanos no se limita a la necesidad de reivindicar las cuestiones que les pertenecen de pleno derecho. Sus relatos expresan una notable preocupación por el bienestar colectivo que se traduce en un elevado interés en animar, ya sea en el plano de las ideas o de las acciones, procesos de transformación social.

En palabras de Asier, hacer visible sus experiencias puede ser un primer paso para cuestionar y mejorar el orden establecido: "La experiencia que yo te he contado, y 
“NOS GUSTARÍA QUE NOS ACOMPAÑASEN EN NUESTRAS DECISIONES”. ALGUNAS CUESTIONES QUE PREOCUPAN A PERSONAS ADULTAS CON DISCAPACIDAD INTELECTUAL SUSANA ROJAS PERNIA E IGNACIO HAYA SALMÓN

también para ver si se pueden pues las cosas que sean malas pues hacerlas, intentar que sean mejores y las que son mejores pues intentar que sean mejores todavía” (19 años).

\section{Discusión de resultados y conclusiones}

Este trabajo parte de la consideración de las personas con discapacidad intelectual como ciudadanos de pleno derecho. De acuerdo con Etxeberría (2008), ciudadano pleno es "[...] la persona a la que se le reconoce el derecho y se le garantizan las posibilidades de realizar su autonomía tanto en su dimensión privada-civil, en la que le corresponde gestionar libremente sus proyectos vitales, como en su dimensión público-política, la que supone tener una participación activa en la organización y gestión en justicia de la sociedad" (p. 27). Lo contrario, su ausencia o el no reconocimiento, sea de hecho o de derecho, tiene temibles consecuencias para quienes lo sufren porque sitúa a esas personas en espacios de marginación discriminatorios y limita injustamente lo que son y también lo que pueden llegar a ser.

Los relatos de las dieciséis personas que participaron en la investigación son el testimonio de trayectorias vitales que se caracterizan por lo que significa vivir y estar vivos, por el deseo de ir haciéndose a sí mismos y de ir tomando decisiones en los diferentes contextos en los que viven, se forman, trabajan u ocian. Son dieciséis testimonios personales y, por tanto, únicos que al mismo tiempo expresan en qué medida sus decisiones están condicionadas por circunstancias personales, sociales y económicas, lo que va a incidir en la construcción de una identidad personal y social positiva y posibilitadora.

Sin poder obviar las consecuencias que los recortes en el gasto social, la reducción del apoyo a las familias o la falta de diálogo con las organizaciones de personas con discapacidad tienen sobre las personas con discapacidad intelectual en el acceso a recursos y servicios (Grammenos, 2013), las declaraciones hechas por los participantes nos invitan a reflexionar sobre los modos en los que se forjan los destinos de las personas cuya trayectoria vital se construye bajo la mirada deficitaria de los temporalmente válidos (Allué, 2003) y, por tanto, desde lo que estos (familiares, profesionales o instituciones) entienden que es deseable para las personas con discapacidad intelectual. Aunque es ampliamente compartido que la calidad de vida de las personas con discapacidad se compone de los mismos factores y relaciones que son importantes para todas las personas y que esta mejora cuando las personas tienen la posibilidad de participar en las decisiones que les incumben (Schalock, 1996; Cummins, 1997; Verdugo et al., 2013), los relatos muestran que la toma de decisiones personal queda circunscrita solo a determinados ámbitos y es parcialmente facilitada. Es decir, aunque las personas reclaman esos espacios y/o el poder decidir en las cuestiones que les incumben, parece que las elecciones y decisiones son ajenas.

Es posible pensar y afirmar que el problema principal es el hecho de ser pensados por otros (Saletti, 2008) y, consecuentemente, que los proyectos sean los de otros. De alguna manera, los proyectos están supeditados a las valoraciones y directrices que establecen otras personas o instituciones sin reconocer la agencia de cada individuo 


\section{“NOS GUSTARÍA QUE NOS ACOMPAÑASEN EN NUESTRAS DECISIONES”. ALGUNAS CUESTIONES QUE PREOCUPAN A PERSONAS ADULTAS CON DISCAPACIDAD INTELECTUAL SUSANA ROJAS PERNIA E IGNACIO HAYA SALMÓN}

para dedicar los esfuerzos que estime oportunos al logro de metas particulares. En consecuencia, si bien el acceso a algunos derechos básicos, p. e. la educación, parecen estar garantizados, otros se diluyen.

Las situaciones descritas alejan a las personas con discapacidad intelectual de la idea de decisión y control sobre sus vidas, tal y como reconoce el artículo 12 de la Convención Internacional de los derechos de las personas con discapacidad y las sitúa en una situación de indefensión aprendida. No obstante, algunas personas se resisten a aceptar que otras personas controlen sus deseos e intentan poner en marcha sus proyectos, lanzando un mensaje unánime: nos gustaría que nos acompañasen en nuestras decisiones.

Por último, es importante señalar que los resultados instan a continuar desde los planteamientos que ofrece la investigación inclusiva. En este sentido, es preciso crear oportunidades para que las personas con discapacidad intelectual puedan hablar sobre lo que para ellas es importante (Deguara, Jelassi, Micallef y Callus, 2012) y descubrir y reconocer sus necesidades prácticas y culturales (Nind y Vinha, 2012).

\section{Referencias bibliográficas}

AldRIDge, J. (2007). Picture this: the use of participatory photographic research methods with people with learning disabilities. Disability and Society, 22 (1), 1-17. doi: 10.1080/096875906 01056006.

Allué, M. (2003). Discapacitados. La reivindicación de la igualdad en la diferencia. Barcelona: Ediciones Bellaterra.

Воотн, T. у Воотн, W. (1994). Parenting under pressure. Mothers and fathers with learning difficulties. Londres: Open University Press.

Bоотн, T. у Воотн, W. (2003). In the Frame: Photovoice and mothers with learning difficulties. Disability and Society, 18 (4), 431-442. doi: 10.1080/0968759032000080986.

Claro, J. G. y Pecino, R. M. (2015). Calidad de vida en personas con diversidad funcional intelectual del programa "grupos de autogestores": una aproximación desde la escala INICO-FEAPS. Documentos de Trabajo Social: Revista de Trabajo y Acción Social, 55, 72-96.

Colectivo IOÉ (2012). Discapacidad e inclusión social. Colección Estudios Sociales, 33. Barcelona: La Caixa.

CSIC (2011). Código de Buenas Prácticas Científicas del CSIC. Madrid: Consejo Superior de Investigaciones Científicas.

Cummins, R. A. (1997). Assessing quality of life. En R. I. Brown (Comp.), Quality of life for people with disabilities (pp. 116-150). United Kingdom: Stanley Thornes Ltd.

Deguara, M., Jelassi, O., Micallef, B. y Callus, A. M. (2012). How we like to live when we have the chance. British Journal of Learning Disabilities, 40, 123-127. doi: 10.1111/j.14683156.2012.00743.x

Denzin, N. y Lincoln, I. (2012). Manual de investigación cualitativa. Barcelona: Gedisa.

ETXEBERRIA, X. (2008). La condición de ciudadania de las personas con discapacidad intelectual. Bilbao: Universidad de Deusto.

EtXeberRía, X. (2016). Autonomía y decisiones de representación/sustitución en personas con discapacidad intelectual. Perspectiva ética. Siglo Cero, 47 (1), 55-66. doi: http://dx.doi. org/1014201/scero201615566. 


\section{“NOS GUSTARÍA QUE NOS ACOMPAÑASEN EN NUESTRAS DECISIONES”. ALGUNAS CUESTIONES QUE PREOCUPAN A PERSONAS ADULTAS CON DISCAPACIDAD INTELECTUAL SUSANA ROJAS PERNIA E IGNACIO HAYA SALMÓN}

Fernández Cordero, L. (2013). El reto de la Vida Independiente. La experiencia de la Fundación Síndrome de Down del País Vasco en la prestación de apoyos a Personas con Discapacidad Intelectual. Revista Española de Discapacidad, 1 (2), 159-165.

Fullana, J., Pallisera, M., Vilà, M. y Puyalto, C. (2016). Las personas con discapacidad intelectual como investigadoras. Debates, retos y posibilidades de la investigación inclusiva. Empiria. Revista de Metodología de Ciencias Sociales, 33, 111-138. doi: http://dx.doi. org/10.5944/empiria.33.2016.15866.

García Muñoz, O. (2011). Lectura fácil: métodos de redacción y evaluación. Madrid: Real Patronato sobre Discapacidad.

GibBs, G. (2012). El análisis de datos cualitativos en investigación cualitativa. Madrid: Morata.

Gilbert, T. (2004). Involving people with learning disabilities in research: issues and possibilities. Health and Social Care in the Community, 12 (4), 298-308. doi: 10.1111/j.13652524.2004.00499.x.

Goodley, D. (2000). Self-advocacy in the lives of people with learning difficulties. The politics of resilience. Buckingham: Open University Press.

Grammenos, S. (2013). European comparative data on Europe 2010 E People with disabilities. Final Report. Academic Network of European Disability Experts (ANED). Recuperado el 3 de junio de 2016, http://digitalcommons.ilr.cornell.edu/cgi/viewcontent.cgi?article=156 98context $=$ gladnetcollect.

Hammersley, M. y Atkinson, P. (2009). Etnografía. Métodos de investigación. Barcelona: Paidós.

Haya, I., Rojas, S. y Lázaro, S. (2014). Observaciones metodológicas sobre la investigación inclusiva: "Me gustaría que sacarais que la persona con discapacidad tiene su propio pensamiento". Revista de Investigación en Educación, 12 (2), 135-144.

InClusion InTERNATIONAL (2014). Independent but not alone. Global report on the right to decide. Recuperado de http://inclusion-international.org/wp-content/uploads/2014/06/ Independent-But-Not-Alone.pdf.

KuckarTZ, U. (2007). Realizing Mixed-Methods Approaches with MAXQDA. Recuperado de http://maxqda.com/download/MixMethMAXQDA-Nov01-2010.pdf.

Kvale, S. (2011). Las entrevistas en investigación cualitativa. Madrid: Morata.

MEC (2015). Datos y cifras. Curso escolar 2015/2016. Madrid: Secretaría General Técnica.

Nind, M. y VINHA, H. (2012). Doing research inclusively: bridges to multiple possibilities in inclusive research. British Journal of Learning Disabilities, 42, 102-109. doi: 10.1111/bld.12013.

Nind, M. (2014). What is inclusive research? London: Bloomsbury.

ONU (2006). Convención Internacional de los Derechos de las personas con discapacidad. Ginebra: Asamblea General de las Naciones Unidas.

Pallisera, M. y Puyalto, C. (2014). La voz de las personas con discapacidad intelectual en la investigación: hacia el desarrollo de investigaciones inclusivas. Revista Nacional e Internacional de Educación Inclusiva, 7 (2), 84-97.

Pérez GiL, R. (2007). Grupos de autogestores en FEAPS: 10 años de experiencia. Personas con discapacidad intelectual se expresan y deciden por sí mismas. Revista de Servicios Sociales y Política Social, 80, 37-61.

Puyalto, C. (2016). La vida independiente de las personas con discapacidad intelectual. Análisis de los apoyos y las barreras que inciden en la consecución de sus proyectos de vida. Tesis doctoral.

RODRígUEZ, G. (Ed.) (2012). El sector de la discapacidad: realidad, necesidades y retos futuros. Análisis de la situación de la población con discapacidad y de las entidades del movimiento asociativo y aproximación a sus retos y necesidades en el horizonte 2020. Madrid: Colección CERMI. 


\section{“NOS GUSTARÍA QUE NOS ACOMPAÑASEN EN NUESTRAS DECISIONES”. ALGUNAS CUESTIONES QUE PREOCUPAN A PERSONAS ADULTAS CON DISCAPACIDAD INTELECTUAL SUSANA ROJAS PERNIA Y IGNACIO HAYA SALMÓN}

Rojas, S., Susinos, T. y Calvo, A. (2013). 'Giving voice' in research processes: an inclusive methodology for researching into social exclusion in Spain. International Journal of Inclusive Education, 17 (2), 156-173. doi: http://dx.doi.org/10.1080/13603116.2011.629687.

RomañaCH, J. (2011). Asistencia personal: la prestación económica para la autonomía y la vida independiente. Papeles de Economía Española, 129, 207-223.

Saletti, L. (2008). Propuestas teóricas feministas en relación al concepto de maternidad. Clepsydra, 7, 169-183.

Shakespeare, T., Davies, D. y Gillespie-Sells, K. (1996). Sexual politics of disability: untold desires. Londres: Cassell.

Schalock, R. L. (1996). Reconsidering the conceptualization and measurement of quality of life. En R. L. Shalock y G. N. SipersteIn (Eds.), Quality of life: Conceptualization and Measurement, 1 (pp. 123-139). USA: AAMR.

TAMARIT, J. y EsPejo, L. (2013). Experiencias de empoderamiento de personas con discapacidad intelectual o del desarrollo. Siglo Cero, 44 (2), 22-35.

Toboso-Martín, M. (2013). De los discursos actuales sobre la discapacidad en España. Política y Sociedad, 50 (2), 681-706. doi: http://dx.doi.org/10.5209/rev_POSO.2013.v50.n2.39007.

Trias, K. (Dir.) (2015). Fundació Catalana Sindrome de Down. Servicios y metodología, 5. ${ }^{\text {e ed. }}$ Barcelona: FCSD.

Verdugo, M. Á., Gómez Sánchez, L., Arias, B., Santamaría, M., Clavero, D. y Tamarit, J. (2013). Evaluación de la calidad de vida en personas con discapacidades intelectuales o del desarrollo: la escala inicio-Feaps. Siglo Cero, 44 (3), 6-20.

WALMSLEy, J. (2004a). Inclusive learning disability research: the (nondisabled) researcher's role. British Journal of Learning Disabilities, 32, 65-71. doi: 10.1111/j.1468-3156.2004.00281.x.

Walmsley, J. (2004b). Involving users with learning difficulties in health improvement: lessons from inclusive learning disability research. Nursing Inquiry, 11 (1), 54-64. doi: 10.1111/j.14401800.2004.00197.x.

Webster, L. y Mertova, P. (2007). Using narrative inquiry as a research method. Abingdon: Routledge.

Williams, V. (2000). Investigando juntos. Siglo Cero, 31, 191, 5-9.

\section{Agradecimientos}

El trabajo de investigación ha sido posible gracias a la participación de jóvenes con discapacidad intelectual de dos entidades Plena Inclusión y l'Institut Municipal d’Educació i Treball. 